Revista EIA, ISSN 1794-1237 Número 8, p. 147-163. Diciembre 2007

Escuela de Ingeniería de Antioquia, Medellín (Colombia)

\title{
APROXIMACIÓN A LA REPRESENTACIÓN EN XML DE OBJETOS DICOM PARA FOTOGRAFİA MÉDICA DIGITAL
}

\author{
CARLOS Ruiz* \\ ANDRÉS TRUJILLO** \\ Alvin GarCíA***
}

\section{RESUMEN}

El estándar DICOM (Digital Imaging and Communication in Medicine) es un protocolo no propietario para el intercambio de información médica. DICOM representa y define la información de objetos del mundo real tales como una resonancia magnética (MRI), una tomografía computarizada (CT) y una fotografía médica digital (VL Photographic), por medio de definiciones de objeto de información llamados IOD. El presente artículo describe una metodología para representar el IOD de una fotografía médica digital de luz visible (VL Photographic Image) por intermedio de documentos XML Schema. Estos documentos se utilizan en la creación y validación de documentos XML para representar información clínica técnica asociada a fotografías médicas digitales para su posterior implementación en una aplicación web de teledermatología.

PALABRAS CLAVE: DICOM; XML Schema; fotografía médica digital; dermatología.

\section{ABSTRACT}

DICOM standard (Digital Imaging and Communication in Medicine) is a non-proprietary protocol for the medical exchange information. DICOM represents and defines the information of real world objects

* Ingeniero Biomédico. Joven Investigador Colciencias-EIA-Universidad CES, Investigador Grupo de Investigación de Ingeniería Biomédica EIA-CES (Gibec). cruiz@eia.edu.co

** Médico y MBA. Director, Instituto Colombiano Medicina Tropical. Coordinador Programa Ingeniería Biomédica EIA-Universidad CES y Profesor Universidad CES. atrujillo@ces.edu.co

* * Z Zootecnista, Candidato a Magíster en Ingeniería Informática. Grupo de Investigación en Bioingeniería (GIB) Universidad CES-Universidad EAFIT y Profesor Instructor Universidad CES. agarcia@ces.edu.co

Artículo recibido 15-IX-2007. Aprobado 19-XI-2007

Discusión abierta hasta junio de 2008 
like a magnetic resonance image (MRI), a computerized axial tomography and a digital medical photography (VL photographic), through information object definitions called IOD. The present article describes a methodology to represent the IOD of a digital medical photography of visible light (VL Photographic Image) through XML Schema documents. These documents were used in the creation and validation of XML documents to represent digital medical photographies compiling clinical and technical information for their later implementation in a teledermatology application.

KEY WORDS: DICOM; XML Schema; digital medical photography; dermatology.

\section{INTRODUCCIÓN}

El estándar DICOM (Digital Imaging and Communications in Medicine $)^{1}$ es un protocolo no propietario para el intercambio de información médica. Define un formato de imagen digital y una estructura de archivo para las imágenes e información asociada². DICOM específicamente describe: i) el contenido de la información, incluyendo la estructura y codificación; ii) servicios DICOM para la administración de la información; y iii) protocolo de mensajería ${ }^{3}$. En la actualidad, es ampliamente usado en diversas especialidades médicas como la radiología, cardiología y oncología. Desde 1983 , DICOM se ha convertido en un estándar aplicable a una gran variedad de sistemas médicos que permite producir, almacenar, visualizar, procesar, enviar, recuperar, buscar e imprimir imágenes médicas y documentos estructurados derivados, así como el manejo de volumen de trabajo ${ }^{4}$.

DICOM tomó mayor fuerza en otras especialidades médicas tales como la endoscopia, la oftalmología y la dermatología, debido al desarrollo y publicación de la estructura de la "definición de objeto de información" (IOD Information Object Definition" ${ }^{* 5,6}$ para imágenes de luz visible de un solo cuadro (Visible Light Single-Frame Image) que soportan las imágenes de color o las monocromáticas producidas por endoscopios, microscopios y cámaras fotográficas. Con esta evolución se abrió la puerta para que diversos sistemas de información en especialidades como endoscopia, patología y dermatología se desarrollasen cumpliendo con ese estándar mundialmente aceptado.

Dentro de la comunidad clínica se ha reconocido la necesidad de utilizar estándares de información en todos los aspectos de la consulta médica. En el caso dermatológico, la información técnica relacionada con la adquisición de fotografías médicas debe ser gestionada de forma adecuada con el fin de brindarle al personal clínico una mayor calidad en tratamiento de las imágenes de estudio ${ }^{7}$. Con respecto a esto, DICOM define la estructura de información necesaria por medio de IOD para integrar información clínica estructurada junto con fotografías médicas digitales. Además, brinda la oportunidad de utilizar herramientas de desarrollo open source y comerciales que facilitan el desarrollo e interoperabilidad de aplicaciones en informática médica con un estándar no propietario ${ }^{7}$.

Una de las herramientas de mayor uso actual para la creación de documentos que contienen información estructurada es XML (Extensible Markup Language). XML es un lenguaje metasintaxis, derivado de SGML (Standard Generalizad Markup Language) diseñado para crear nuevos lenguajes, describir información con sus respectivas estructuras, con el fin de transferirse a través de redes (Internet) y ser procesada por el usuario final ${ }^{8,9}$. En los últimos años, DICOM ha

* Una Definición de Objeto de Información (Information Object Definition IOD) es un modelo de datos abstractos orientado a objetos utilizado para especificar información sobre objetos del mundo real. Un IOD proporciona comunicación de entidades aplicativas con un "vistazo general" de la información para ser intercambiada. 
seguido los pasos de HL7 (Health Level 7) ${ }^{10}$ incursionando en XML por medio de sus grupos de trabajo (WG). Específicamente, el WG-06 (Base Standard) ha trabajado en el desarrollo de mecanismos para la publicación del estándar DICOM en XML. Por su parte, la hoja de ruta del WG-10 (Strategic Advisory) está encaminada a explorar y dirigir la evolución del SR DICOM en relación con el progreso de CDA (Clinical Document Architecture) de HL7 y desarrollos web en donde se encuentra XML. Aunque este trabajo ha sido extenso y tiene el objetivo de desarrollar un modelo de información compartido para trabajar con otros estándares que utilizan otras tecnologías, la creación de una estructura para representar el estándar DICOM en XML no se ha completado a la fecha, mucho menos la generación de un comunicado oficial sobre su aplicación ${ }^{11}$.

Lo anterior no ha sido un impedimento para que diversos trabajos y una patente se hayan desarrollado, con el fin de representar partes del estándar DICOM en documentos XML utilizando el lenguaje derivado XML Schema ${ }^{2,9,12-14}$. Estos trabajos se enfocan en la representación de reportes estructurados (SR) definidos por DICOM, facilitando la recolección y muestra de esta información. Pero a su vez brinda las pautas para la representación de la totalidad del estándar.

Lo anterior, sumado al auge y perfeccionamiento de la fotografía digital, brinda el potencial para el desarrollo de sistemas de información confiables basados en estándares y tecnologías de amplia aceptación, de fácil manejo y aplicación, adaptables a sistemas de almacenamiento de imágenes y comunicaciones (PACS Picture Archiving and Communication Systems). A su vez, la transmisión de esta información a través de redes de telecomunicación electrónicas extensamente accesibles como Internet permiten un mejor servicio y acceso a la información ${ }^{3}$.
En este artículo se describen los desarrollos en XML Schema que buscan representar objetos DICOM, específicamente el VL Photographic IOD, que servirá como modelo de datos para la recopilación de información clínica y fotográfica para su posterior implementación en un sistema de teledermatología, aplicando el estándar DICOM con el uso de una plataforma de gestión de imágenes vía web ${ }^{15}$.

La organización del presente artículo es la siguiente. En la primera sección, se brinda una breve introducción a la estructura de datos utilizada por el estándar DICOM y a las aproximaciones para representarlo mediante XML Schema. En la siguiente sección, se apreciarán las representaciones gráficas e interpretación de los resultados. Por último, se analiza el trabajo desarrollado y se proponen conclusiones y sugerencias para trabajos futuros.

\section{ESTÁNDAR DICOM Y METODOLOGÍA DE REPRESENTACIÓN EN XML SCHEMA}

\subsection{Estructura del IOD DICOM}

El componente de información de DICOM se representa por medio de "definiciones de objeto de información” denominados IOD. Estos IOD representan entidades del mundo real, como una tomografía computarizada (CT), una resonancia magnética $(\mathrm{RM})$, una fotografía digital o un reporte estructurado. Los IOD están integrados por componentes o entidades de información (IE). En DICOM se definen 17 IE para describir información del paciente, estudios, series, equipos, imágenes, entre las más importantes. Así mismo cada IE está integrada por diversos atributos de información llamados módulos. Para el desarrollo de este trabajo se enfocó en representar el VL fotográfico IOD (tabla 1). 
Tabla 1. VL Fotográfico IOD. Fuente: Estándar DICOM PS 3-2007 [5]

\begin{tabular}{|l|l|l|l|}
\hline \multicolumn{1}{|c|}{ IE } & \multicolumn{1}{|c|}{ Module } & Reference & \multicolumn{1}{c|}{ Usage } \\
\hline Patient & Patient & C.7.1.1 & $\mathrm{M}$ \\
\hline & Specimen Identification & C.7.1.2 & $\begin{array}{l}\text { C - Required if the Imaging Subject } \\
\text { is a Specimen }\end{array}$ \\
\hline & Clinical Trial Subject & $\mathrm{C} .7 .1 .3$ & $\mathrm{U}$ \\
\hline Study & General Study & $\mathrm{C} .7 .2 .1$ & $\mathrm{M}$ \\
\hline & Patient Study & $\mathrm{C} .7 .2 .2$ & $\mathrm{U}$ \\
\hline & Clinical Trial Study & $\mathrm{C} .7 .2 .3$ & $\mathrm{U}$ \\
\hline Series & General Series & $\mathrm{C} .7 .3 .1$ & $\mathrm{M}$ \\
\hline & Clinical Trial Series & $\mathrm{C} .7 .3 .2$ & $\mathrm{U}$ \\
\hline Equipment & General Equipment & $\mathrm{C} .7 .5 .1$ & $\mathrm{M}$ \\
\hline Image & General Image & $\mathrm{C} .7 .6 .1$ & $\mathrm{M}$ \\
\hline & Image Pixel & $\mathrm{C} .7 .6 .3$ & $\mathrm{M}$ \\
\hline & Acquisition Context & $\mathrm{C} .7 .6 .14$ & $\mathrm{M}$ \\
\hline & Device & $\mathrm{C} .7 .6 .12$ & $\mathrm{U}$ \\
\hline & VL Image & $\mathrm{C} .8 .12 .1$ & $\mathrm{M}$ \\
\hline & Overlay Plane & $\mathrm{C} .9 .2$ & $\mathrm{U}$ \\
\hline & SOP Common & $\mathrm{C} .12 .1$ & $\mathrm{M}$ \\
\hline
\end{tabular}

\subsubsection{VL fotográfico IOD}

El VL fotográfico IOD especifica los atributos de información para una imagen fotográfica de luz visible de un solo cuadro o fotografía médica digital. Está compuesto por 5 IE y 16 módulos entre obligatorios $(\mathrm{M})$, condicionales $(\mathrm{C})$ y opcionales (U). El IE paciente (Patient IE) describe e identifica al paciente que es sujeto del estudio diagnóstico. El IE Estudio (Study IE) describe los atributos de información del paciente y estudio necesarios para la interpretación diagnóstica de la imagen. El IE Series (Series IE) especifica los atributos de información que identifican y describen la información general sobre la serie de instancias compuestas agrupadas dentro de un solo estudio. El IE equipo (Equipment IE) describe el dispositivo que produce la serie de instancias compuestas y, por último, el IE Imagen (Image IE) identifica y describe la imagen dentro de una serie particular. De esta manera, el estándar DICOM promueve la calidad e integridad de los datos por medio de la unión de importante información estructurada sobre la imagen junto con la imagen como tal.

\subsection{Metodología para la representación de VL fotográfico IOD en XML Schema}

En la figura 1 se ilustra un esquema básico utilizado para generar la representación del IOD correspondiente. En primer lugar se debe desarrollar un documento XML Schema correspondiente al diccionario de datos (DICOM Dictionary, en este caso) de conformidad con los atributos de información necesarios para nuestro IOD. En segundo lugar, se representan los módulos y macros obligatorios identificando los atributos de información contenidos en varios módulos. En ese caso los atributos se designan de acuerdo con el menor tipo, a menos que su descripción indique lo contrario. A continuación se generan los documentos XML Schemas pertenecientes a los IE comunes para nuestro IOD. Por último, estos IE se integran en un documento general que contiene el diccionario de datos y los macros para formar el documento XML Schema del IOD. 


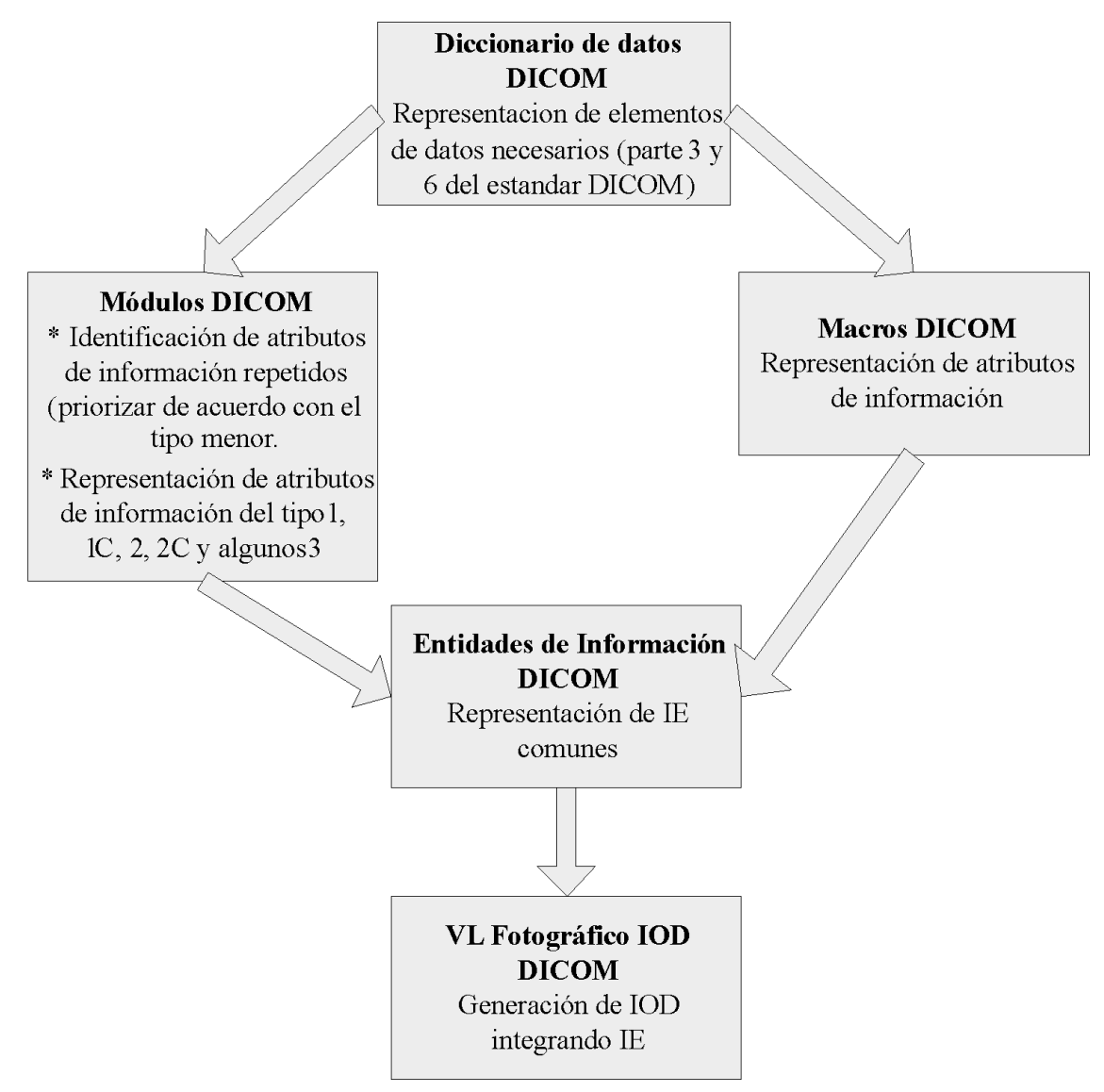

Figura 1. Esquema básico para la presentación de VL fotográfico IOD

A partir del documento XML Schema, se genera un archivo XML que se utiliza como plantilla de recopilación de información en formato XHTML por medio de transformaciones XSLT. Así mismo, los metadatos asociados se ingresan a la base de datos de la aplicación web permitiendo al usuario final visualizar la información, consultar estudios anteriores y establecer búsquedas según atributos específicos de información.

\subsubsection{Atributo de información, elementos de datos}

El estándar DICOM ordena los atributos de información en tablas que poseen especificaciones sobre el uso, tipo y valor de representación de cada atributo de información, así como las secuencias de atributos y macros anidados en las tablas. Los atributos de información de un IOD definido por DICOM describen las propiedades de una instancia de una entidad del mundo real ${ }^{5}$. Tales atributos se codifican como elementos de datos (data element), que son unidades de información que poseen valores codificados para los diferentes atributos del IOD. Estos elementos de datos poseen campos de información que definen su estructura ${ }^{16}$ :

- Etiqueta del elemento de datos (data element tag).

- Representación del valor (value representation VR).

- Campo de valor (value field).

- Valor de multiplicidad (value multiplicity VM). 
Adicionalmente a estos campos, DICOM define en los módulos otras características para los elementos de datos ${ }^{16}$ :

- Tipo de elemento de datos (data element type)

- Valores enumerados (enumerated value)

- Elementos de datos privados (private data element)

- Elementos de dato retirado

\section{Representación de atributo de informa- ción en XML Schema}

XML Schema da la posibilidad de crear elementos con sus respectivos atributos, valores fijos y enumerados. Una representación gráfica del XML Schema del elemento de datos Content Sequence se observa en la figura 2. En la figura 3 aparece la codificación del mismo elemento en XML Schema.

Como se aprecia en la figura 3, los atributos de información de cada elemento de datos se representan de forma grupal por medio del elemento de XML Schema "attribute Group". Estos grupos de información se codifican en un documento XML Schema llamado "DICOMdictionary.xsd", que per-

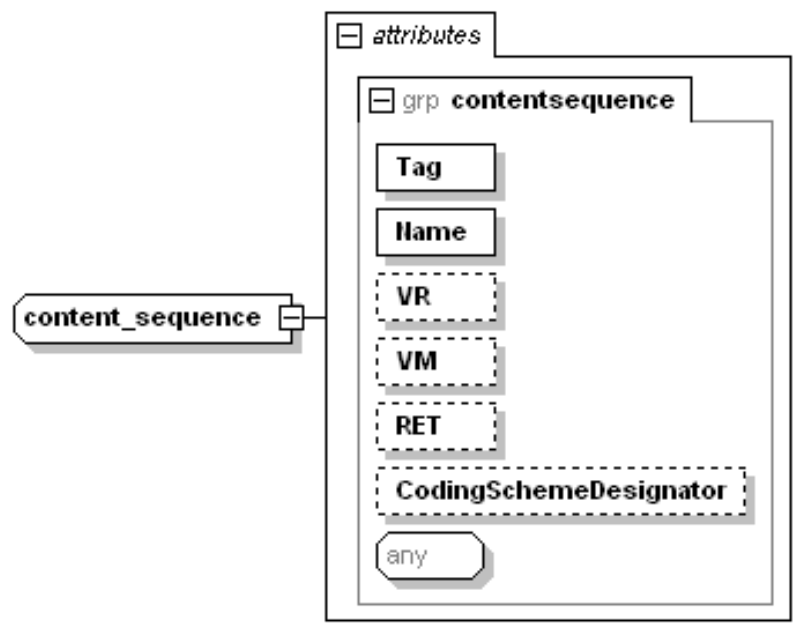

Generated by XmISpy

www.altova.com

Figura 2. Representación gráfica de XML Schema para el elemento Content Sequence

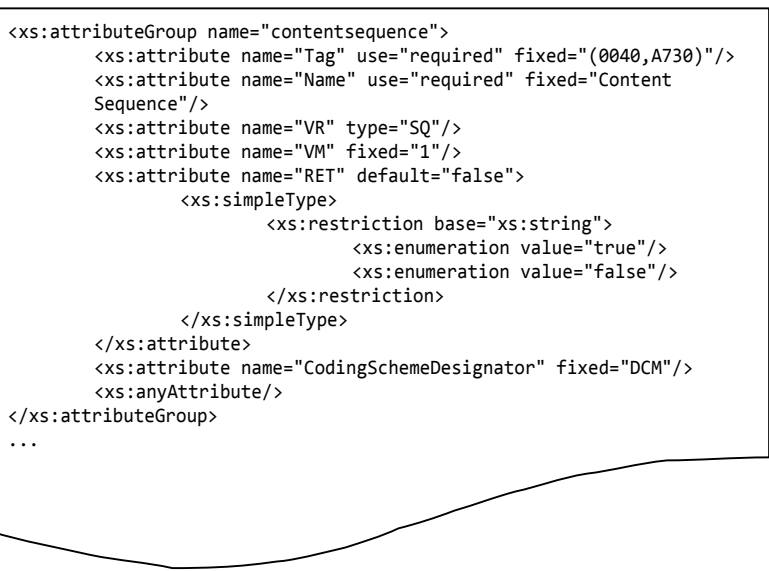

Figura 3. Codificación XML Schema para elementos de datos Content Sequence

mite estructurar modularmente los documentos, insertando diversos XML Schemas dentro de otros.

Por su parte el atributo "anyAttribute" permite extender un documento XML instancia proveniente del XML Schema, con atributos no especificados anteriormente.

\subsubsection{Restricciones para atributos de información}

Todos los atributos de información definidos en DICOM poseen restricciones con respecto a sus valores. Antes se describe la representación del valor (value representation VR) definido por DICOM. Estas restricciones definen la estructura del valor del atributo de información. Específicamente, definen la lista de caracteres que se pueden utilizar, así como la longitud de su valor. Adicionalmente a estas restricciones, DICOM define en los módulos los valores definidos o enumerados para algunos atributos de información.

\subsubsection{Restricciones en la representación del valor}

Para las representaciones de valor XML Schema provee mecanismos para representar estas restricciones por medio de su lenguaje natural. Para la siguiente representación definida en la parte 5 del estándar DICOM ${ }^{16}$ : IS (cadena de caracteres de 
entero) su codificación se desarrolla de la siguiente manera.

- IS: Es una cadena de caracteres que representan un número entero. Su lista de caracteres está limitada a los números de 0 hasta 9 y los símbolos "+" y "-" con una longitud máxima de 12 bytes. Su representación en XML Schema se presenta en la figura 4.

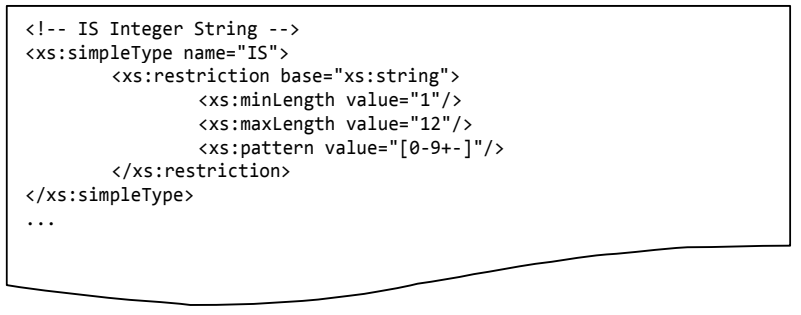

Figura 4. Codificación XML Schema de la representación de valor IS

\subsubsection{Restricciones en valores enumerados}

Diversos atributos de información poseen valores enumerados. Es el caso del atributo de información Patient's Sex, el cual solo puede tomar los valores de $\mathrm{M}$ (masculino), F (femenino) u O (otro). Su representación en XML Schema se presenta en la figura 5.

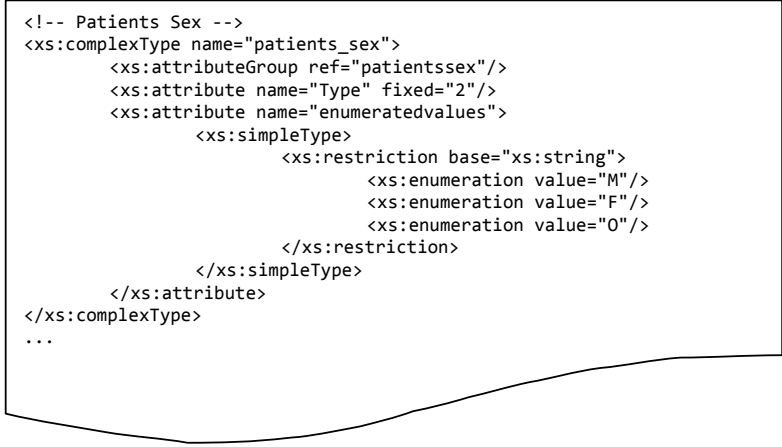

Figura 5. Codificación XML Schema del atributo de información Patient's Sex

\subsubsection{Atributos de información en secuencias}

En ciertas tablas de los IOD se describen secuencias de atributos de información llamadas secuencias de ítems. Estas secuencias se representan por medio del símbolo ">", el cual precede al ítem de información de la secuencia. En la tabla 2 se observa un ejemplo de la estructura de un módulo de información de DICOM con secuencias.

En la figura 6 se observa la representación gráfica del XML Schema del elemento Specimen Sequence, que posee una secuencia de ítems.

Tabla 2. Módulo Specimen Identification. Fuente: Estándar DICOM PS 3-2007 [5]

\begin{tabular}{|c|c|c|c|}
\hline Attribute Name & Tag & Type & Attribute Description \\
\hline Specimen Accession Number & $(0040,050 A)$ & 1 & $\begin{array}{l}\text { A departmental Information System identifier that identifies } \\
\text { the Accession. See Section C.7.1.2.1.1 for further expla- } \\
\text { nation. }\end{array}$ \\
\hline Specimen Sequence & $(0040,0550)$ & 2 & $\begin{array}{l}\text { Detailed description of one or more specimens. Zero or } \\
\text { more Items may be included in this Sequence. }\end{array}$ \\
\hline >Specimen Identifier & $(0040,0551)$ & $2 \mathrm{C}$ & $\begin{array}{l}\text { A departmental information system identifier for the Spe- } \\
\text { cimen. See Section C.7.1.2.1.2 for further explanation. } \\
\text { Required if a sequence item is present. }\end{array}$ \\
\hline $\begin{array}{l}\text { >Specimen Type Code } \\
\text { Sequence }\end{array}$ & $(0040,059 A)$ & $2 \mathrm{C}$ & $\begin{array}{l}\text { Specimen Type. Only a single Item shall be permitted in } \\
\text { this Sequence. Required if a sequence item is present and } \\
\text { Specimen Identifier }(0040,0551) \text { is sent. }\end{array}$ \\
\hline \multicolumn{2}{|c|}{ >>Include ‘Code Sequence Macro' Table 8.8-1 } & \multicolumn{2}{|r|}{ No Baseline Context IDs are defined } \\
\hline >Slide Identifier & $(0040,06 \mathrm{FA})$ & $2 \mathrm{C}$ & $\begin{array}{l}\text { Identifier of the Slide. } \\
\text { Required if a sequence item is present and the Specimen } \\
\text { is a Slide. }\end{array}$ \\
\hline
\end{tabular}




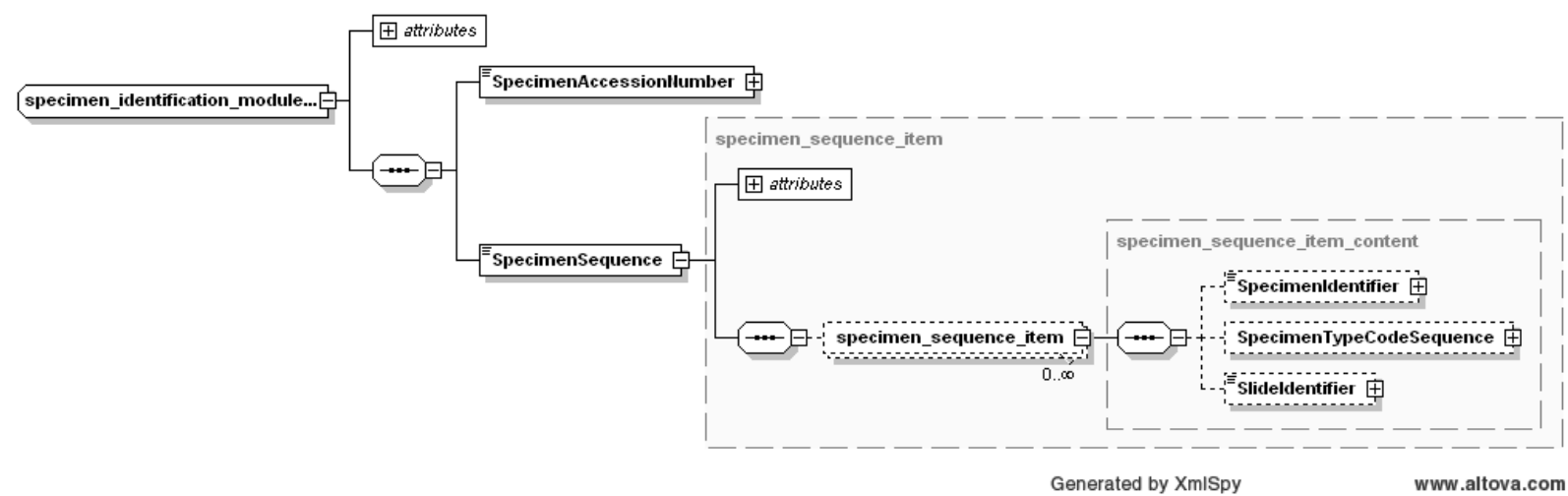

Figura 6. Representación gráfica de XML Schema para la secuencia de ítems del atributo Specimen Sequence

En la figura 7 se presenta la codificación del elemento Specimen Sequence en XML Schema.

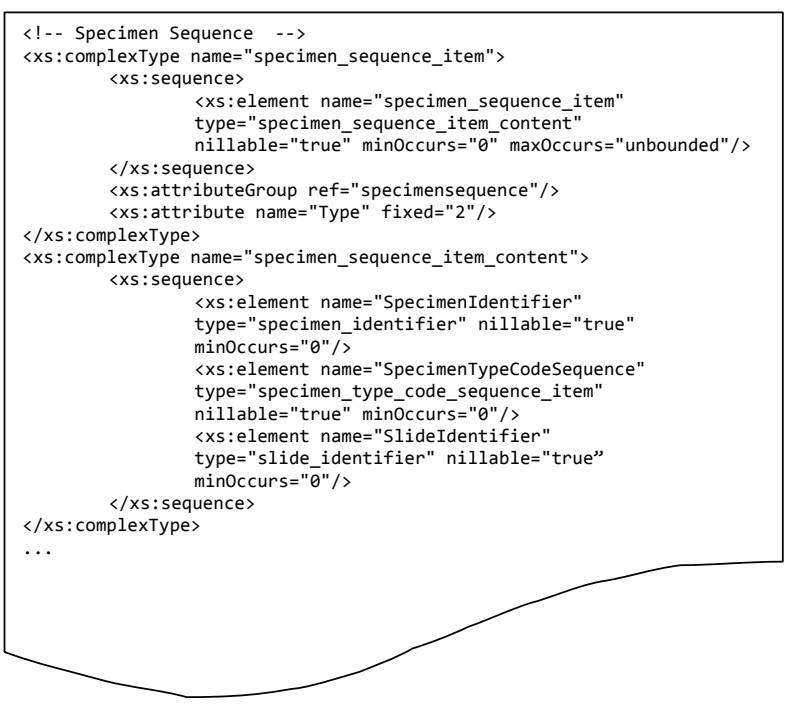

Figura 7. Codificación del XML Schema para la secuencia de ítems del atributo Specimen Sequence

Con respecto a la figura 7, los siguientes puntos de desarrollo deben ser analizados:

- La secuencia de ítems del atributo Specimen Sequence se representa por una secuencia de elementos anidados por medio del Complex Type "specimen_sequence_item", que posee un elemento del mismo nombre que define su con- tenido mediante el Complex Type "specimen_sequence_item_content". Este define la secuencia de ítems anidados por medio del elemento de XML Schema Sequence.

- Como se observa en la descripción del atributo Specimen Sequence en la figura 7, este atributo puede contener uno o más ítems, en donde cada uno de ellos contiene los atributos anidados. Para representar esto se utilizan los atributos "minOccurs" y "maxOccurs". Este último atributo especifica el número máximo de veces que puede presentarse un elemento. Para este caso su valor "unbounded" no define un límite.

\subsubsection{Atributos de información en macros}

En ciertas tablas de módulos, algunos atributos de información hacen referencia a atributos tipo macro que se emplean en casos en los cuales los mismos atributos de información son utilizados en múltiples tablas o en múltiples ubicaciones dentro de un mismo módulo. En la figura 7 se observa el uso del Code Sequence Macro por parte del atributo Specimen Type Code Sequence. En esta ocasión el macro se usa como una secuencia, en donde se insertan los atributos de información de la tabla básica del Code Sequence Macro (tabla 3). 
Tabla 3. Tabla de atributos Macro Code Sequence. Codificación básica. Fuente: Estándar DICOM PS 3-2007 [5]

\begin{tabular}{|l|c|c|l|l|}
\hline \multicolumn{1}{|c|}{ Attribute Name } & Tag & Type & \multicolumn{1}{|c|}{ Attribute Description } \\
\hline Code Value & $(0008,0100)$ & $1 C$ & $\begin{array}{l}\text { See Section 8.1. Required if a sequence item is } \\
\text { present. }\end{array}$ \\
\hline Coding Scheme Designator & $(0008,0102)$ & $1 C$ & $\begin{array}{l}\text { See Section 8.2. Required if a sequence item is } \\
\text { present. }\end{array}$ \\
\hline Coding Scheme Version & $(0008,0103)$ & $1 C$ & $\begin{array}{l}\text { See Section 8.2. Required if a sequence item is present } \\
\text { and the value of Coding Scheme Designator (0008,0102) } \\
\text { is not sufficient to identify the Code Value (0008,0100) } \\
\text { unambiguously. }\end{array}$ \\
\hline Code Meaning & $(0008,0104)$ & $1 C$ & $\begin{array}{l}\text { See Section 8.3. Required if a sequence item is } \\
\text { present. }\end{array}$ \\
\hline$\ldots$ & & & \multicolumn{2}{c}{} \\
\hline
\end{tabular}

\subsubsection{Atributos de información dentro de módulos del IOD}

En las tablas de los módulos de los IOD, DICOM define el tipo del elemento de datos para utilizar, junto con una descripción breve sobre su significado. En la tabla 4 se observa un ejemplo de la estructura de un módulo de información de DICOM. En la figura 8 se observa la representación gráfica del XML Schema de este módulo.

Tabla 4. Módulo del Sujeto de prueba clínica (Clinical Trial Subject.). Fuente: Estándar DICOM PS 3-2007 [5]

\begin{tabular}{|l|c|c|l|}
\hline \multicolumn{1}{|c|}{ Attribute Name } & Tag & Type & \multicolumn{1}{c|}{ Attribute Description } \\
\hline Clinical Trial Sponsor Name & $(0012,0010)$ & 1 & $\begin{array}{l}\text { The name of the clinical trial sponsor. See } \\
\text { C.7.1.3.1.1. }\end{array}$ \\
\hline Clinical Trial Protocol ID & $(0012,0020)$ & 1 & Identifier for the noted protocol. See C.7.1.3.1.2. \\
\hline Clinical Trial Protocol Name & $(0012,0021)$ & 2 & $\begin{array}{l}\text { The name of the clinical trial protocol. See } \\
\text { C.7.1.3.1.3. }\end{array}$ \\
\hline Clinical Trial Site ID & $(0012,0030)$ & 2 & $\begin{array}{l}\text { The identifier of the site responsible for submitting } \\
\text { clinical trial data. See C.7.1.3.1.4. }\end{array}$ \\
\hline Clinical Trial Site Name & $(0012,0031)$ & 2 & $\begin{array}{l}\text { Name of the site responsible for submitting clinical } \\
\text { trial data. See C.7.1.3.1.5 }\end{array}$ \\
\hline Clinical Trial Subject ID & $(0012,0040)$ & $1 \mathrm{C}$ & $\begin{array}{l}\text { The assigned identifier for the clinical trial subject. } \\
\text { See C.7.1.3.1.6. Shall be present if Clinical Trial } \\
\text { Subject Reading ID (0012,0042) is absent. May be } \\
\text { present otherwise. }\end{array}$ \\
\hline Clinical Trial Subject Reading & $(0012,0042)$ & $1 \mathrm{C}$ & $\begin{array}{l}\text { Identifies the subject for blinded evaluations. } \\
\text { Shall be present if Clinical Trial Subject ID } \\
\text { (0012,0040) is absent. May be present otherwise. } \\
\text { See C.7.1.3.1.7. }\end{array}$ \\
\hline
\end{tabular}




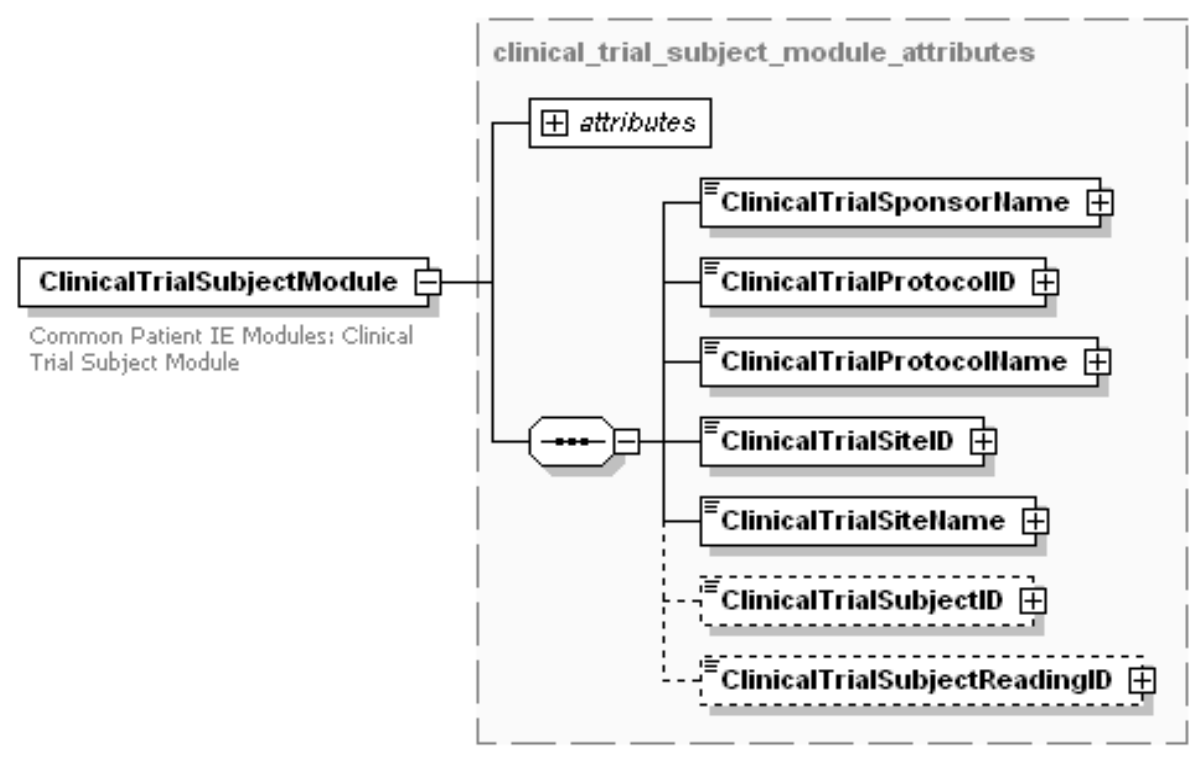

Generated by XmISpy

www.altova.com

Figura 8. Representación gráfica de XML Schema para el módulo Clinical Trial Subject

En la figura 9 se presenta la codificación de tal módulo en XML Schema.

Con respecto a la figura 9, los siguientes puntos de desarrollo deben analizarse:

- Los atributos de información definidos por este módulo se tipifican en 1, 2 y 2C. Esta tipificación se refleja en cada elemento por medio del atributo "nillable". Este atributo del XML Schema especifica si el valor de un elemento puede ser nulo o no. Cuando su valor es "False" (falso), un elemento no puede tener un valor nulo (característico de los elementos 1 y $1 \mathrm{C}$ ); por lo tanto, cuando su valor es "True" (verdadero), un elemento puede tener un valor nulo (característico de los elementos 2, 2C y 3 ).

- Los atributos de información tipificados como 1C y 2C deben ser incluidos en la codificación del estándar con ciertas condiciones. Esto se representa por medio del atributo "minOccurs", que especifica el número mínimo de veces que puede presentarse un elemento. Para los elementos como Clinical Trial Subject Reading ID de la

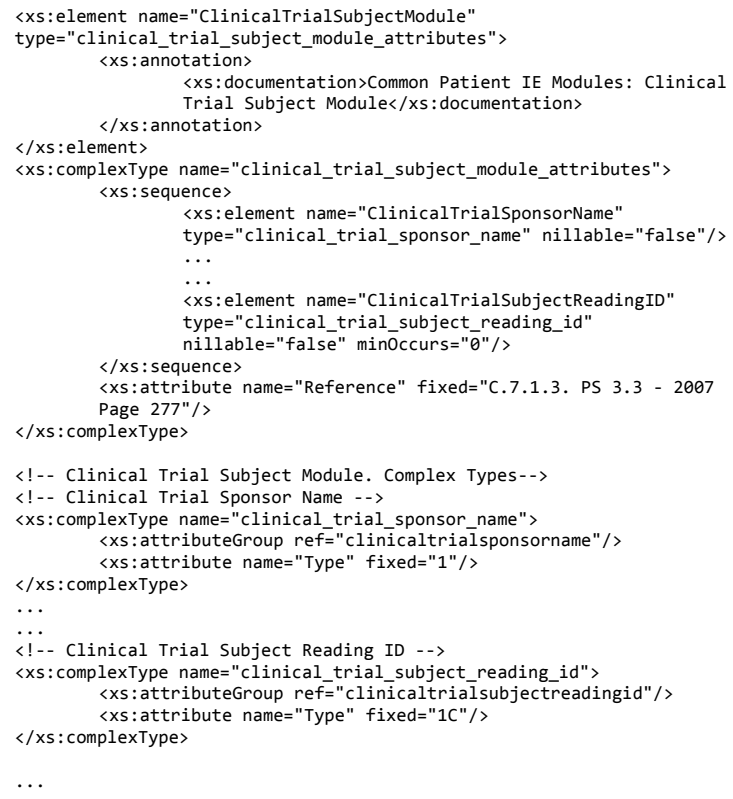

Figura 9. Codificación del XML Schema para el módulo Clinical Trial Subject 
figura 9 se aplica este atributo. Para el resto de los elementos el valor preestablecido de este atributo (1) se aplica.

- Los atributos de información dentro del estándar DICOM pueden aparecer en diferentes tablas con diversos tipos, siendo imposible representar esta característica por medio de un diccionario global ("DICOMdictionary.xsd", en este caso). Por consiguiente, en este desarrollo se define en cada módulo la tipificación de cada elemento.

\subsubsection{Entidades de información}

Las IE están compuestas por los módulos descritos. Son comunes para gran cantidad de IOD, por lo tanto, se denominan Módulos IE Comunes (Common IE Modules). En la figura 10 se observa la representación gráfica del XML Schema para los módulos IE comunes del paciente.

En la figura 11 se presenta la codificación de este IE en XML Schema.

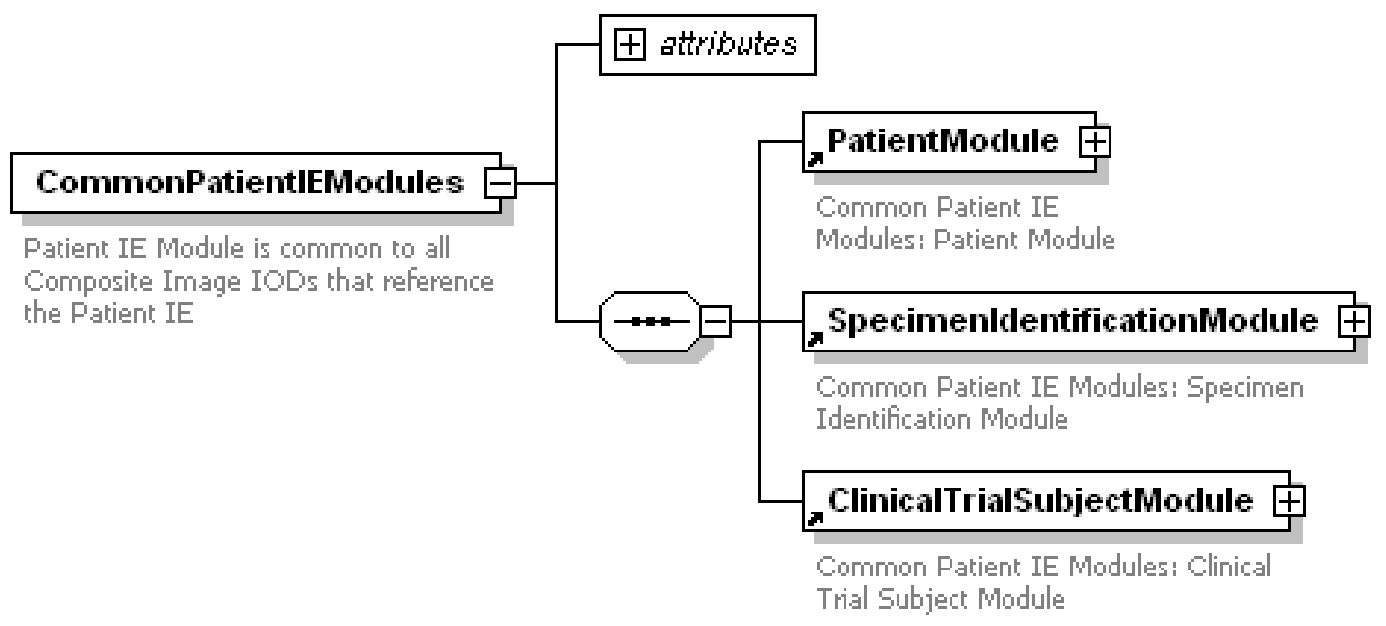

Generated by XmISpy

www.altova.com

Figura 10. Representación gráfica de XML Schema para el módulo Common Patient IE Modules

Con respecto a la figura 11 , el siguiente punto de desarrollo debe analizarse con base en la estructura modular del estándar DICOM, los módulos de información se crean individualmente. A continuación se origina un documento XML Schema que representa cada IE. En estos se hace referencia a los módulos y sus respectivos elementos (xs:element ref="PatientModule").

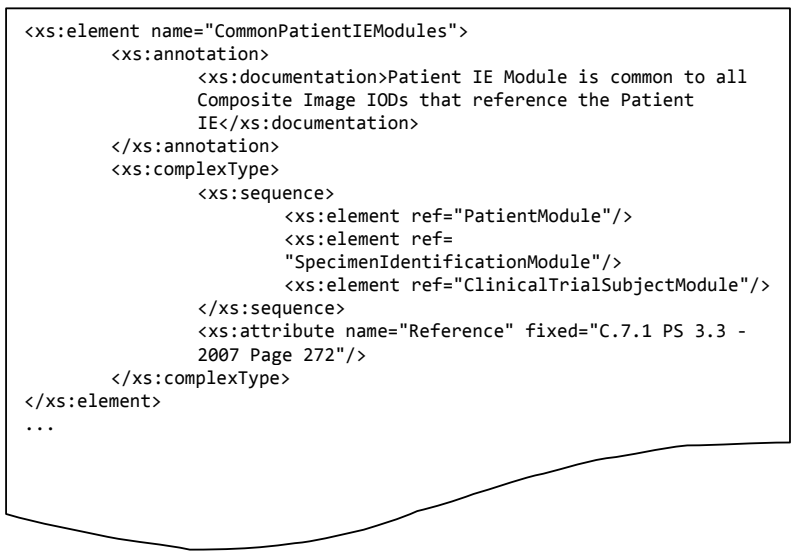

Figura 11. Codificación del XML Schema para el módulo Common Patient IE Modules 
3. REPRESENTACIÓN E IMPLEMENTACIÓN DE VL FOTOGRÁFICO IOD EN XML

\subsection{Representación del VL fotográfico IOD en XML Schema}

En la figura 12 se observa la representación general del XML Schema para el VL fotográfico IOD.

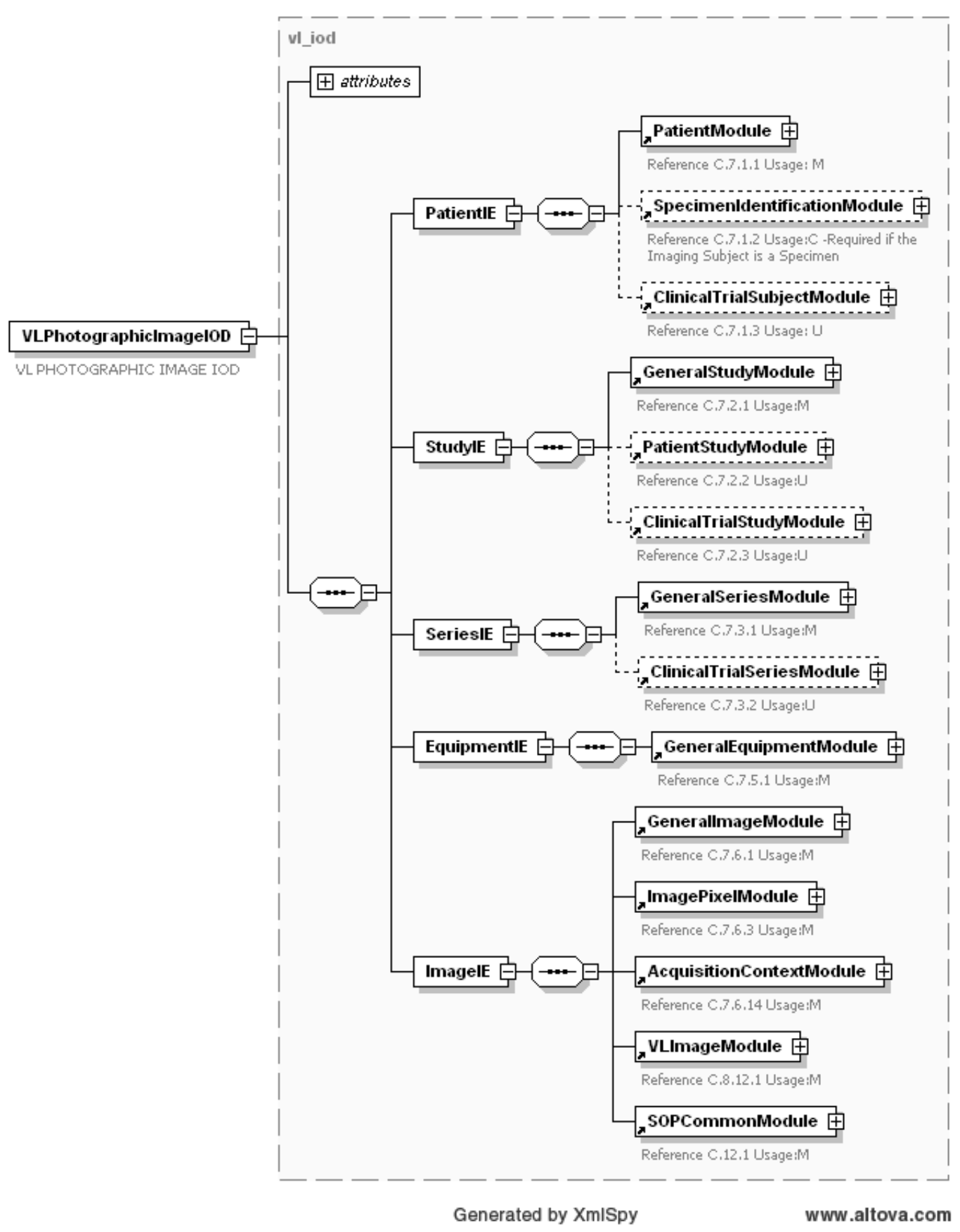

Figura 12. Representación gráfica de XML Schema para el VL fotográfico IOD 
En la figura 13 se presenta la codificación de este IOD en XML Schema.

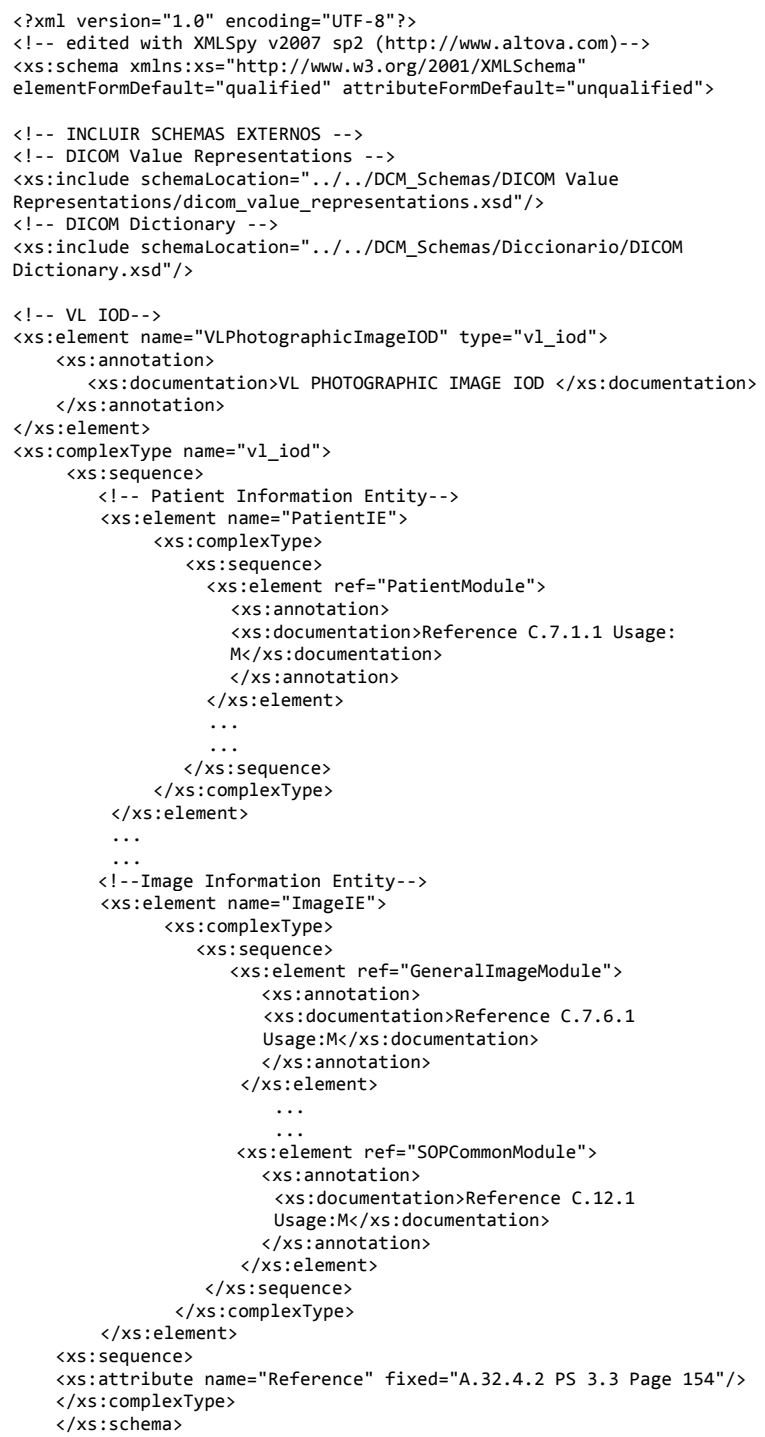

Figura 13. Codificación del XML Schema para el VL fotográfico IOD

\subsection{Implementación del VL fotográfico IOD}

La representación del VL fotográfico IOD en XML Schema permite la flexibilidad para transformar el documento en múltiples formatos diferentes. En primera instancia, un documento XML se obtiene a partir del XML Schema descrito (figura 12). A partir de éste, generamos documentos en formatos XHTML por medio de transformaciones XSLT, necesarios para el ingreso y visualización de la información en la aplicación web. En la figura 14 se observan los pasos de implementación y en la figura 15 se aprecia el proceso en la aplicación web correspondiente ${ }^{15}$.

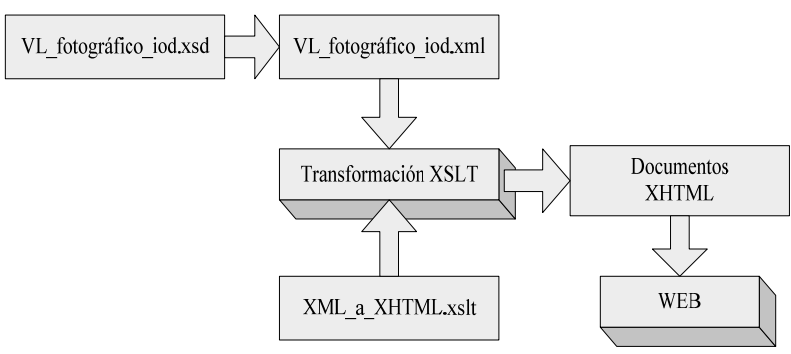

Figura 14. Implementación del VL fotográfico IOD

\subsection{Información clínica recopilada}

Para realizar las pruebas del esquema definido, se tomó la estructura de información de la historia clínica dermatológica definida por el Centro Dermatológico CES Sabaneta. El presente IOD recopila atributos de información comunes con respecto a la información específica del paciente: motivo de consulta, antecedentes personales, revisión por sistemas, entre los más importantes. Además información técnica proveniente de la adquisición de las fotografías: descripción de las imágenes adquiridas, área anatómica de estudio, métodos de compresión, entre otros. Esto permite al IOD adquirir suficiente información, para establecer criterios de búsqueda básicos y avanzados, facilitando al personal dermatológico extraer conocimiento, relacionar estudios y detectar tendencias epidemiológicas. Así mismo, la disposición para intercambiar esta información 
facilita compartir diversos estudios entre personal ca- impulsar el estudio de casos y establecer comunidapacitado, con el fin de obtener segundas opiniones, des virtuales de ayuda en dermatología.

a)

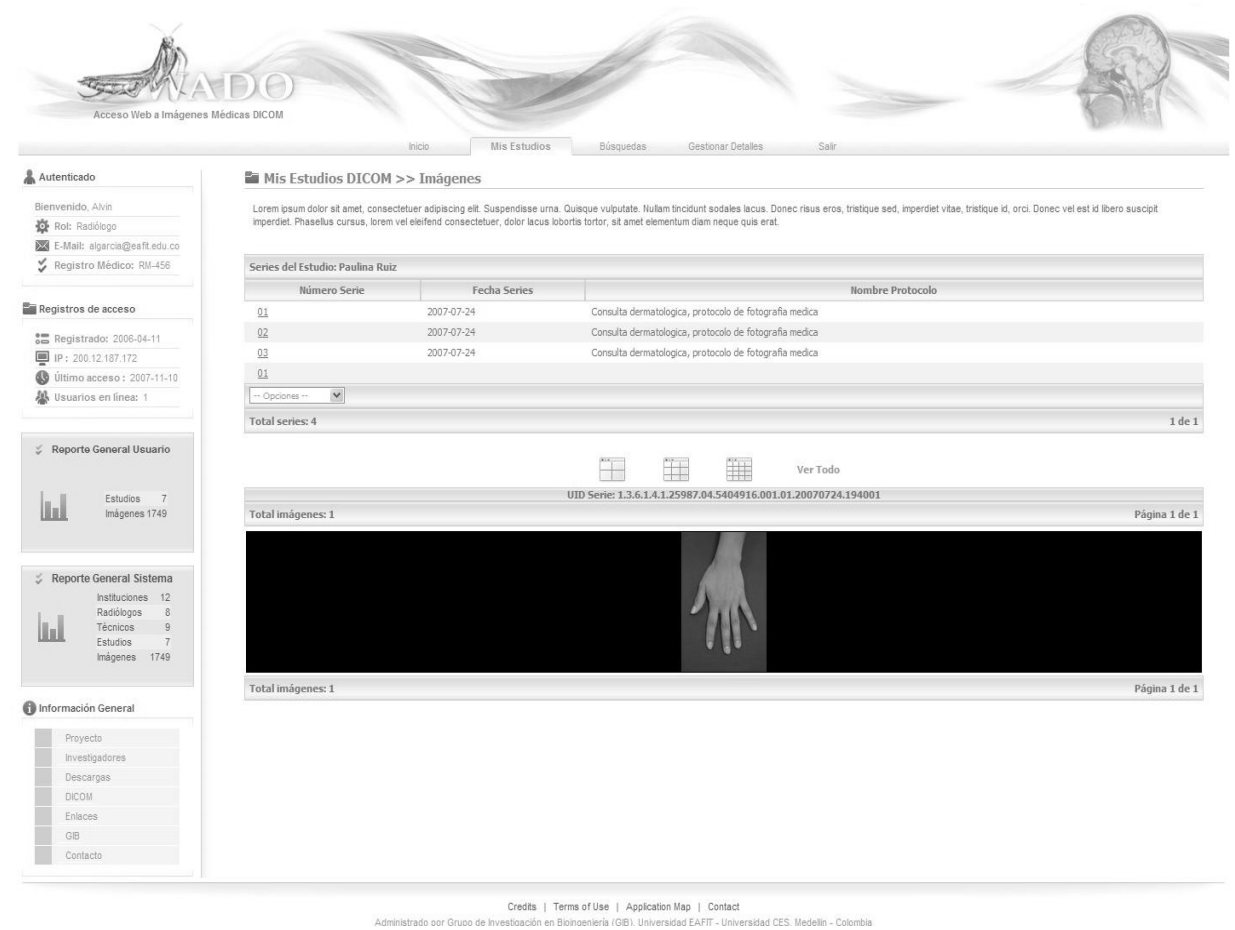

b)

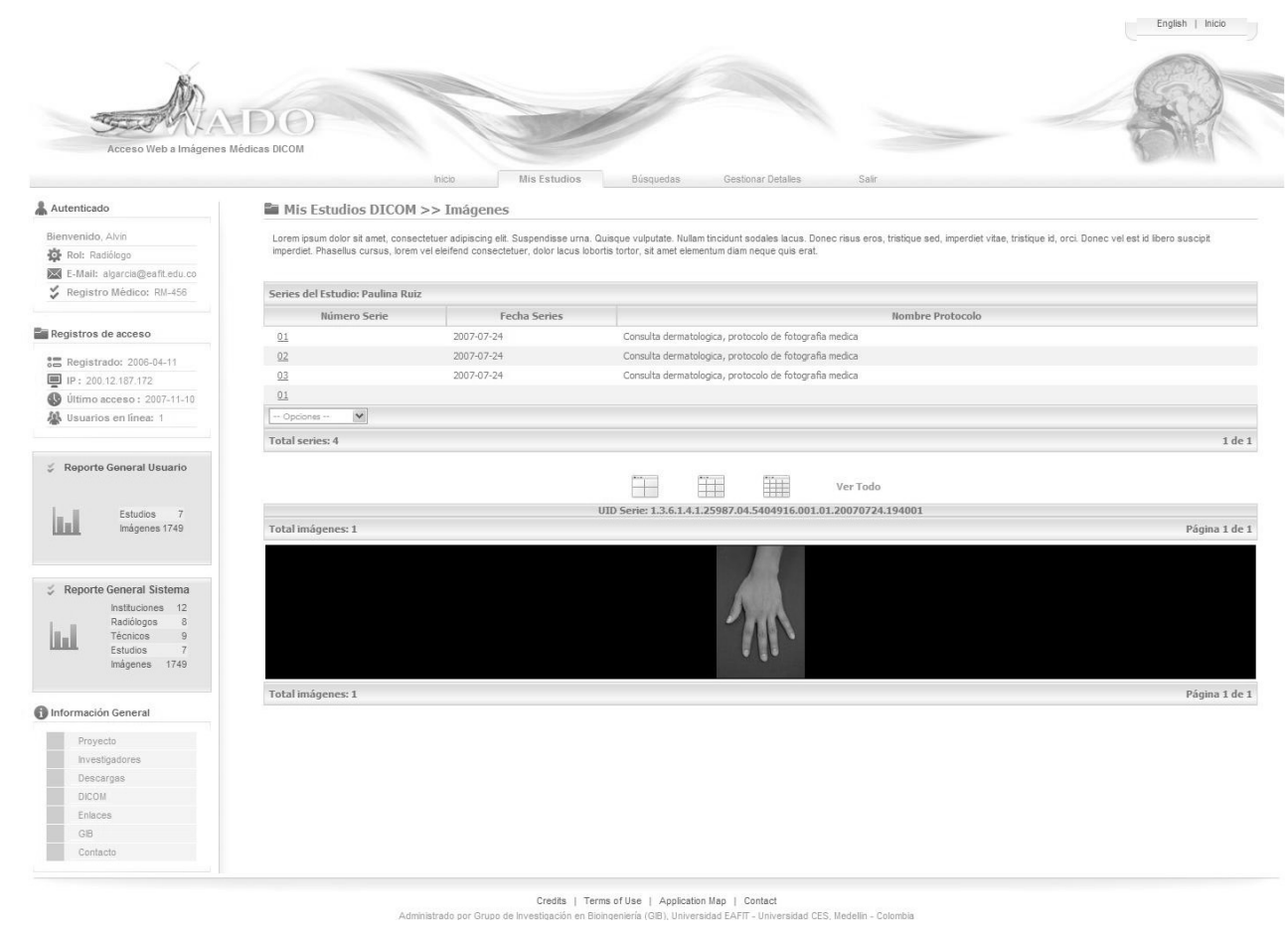


c)

Paulina Ruiz

772323-1

\begin{tabular}{lll}
\hline XC & Modalidad & Imagen \\
\hline
\end{tabular}

Información paciente

Patient Module

PatientsName

PatientID

PatientsBirthDate

PatientsSex

Paulina Ruiz

772323-1

19830114

$\mathrm{F}$

Specimen Identification Module

SpecimenAccessionNumber

SpecimenSequence

1.2.840.1008.1.2.1

1.2.840.10008

Clinical Trial Subject Module

General Study Module

\section{Información estudio}

\section{StudyDate}

20070724

StudyTime

191538

1010

InstitutionName

ReferringPhysiciansName

Centro Dermatológico CES Sabaneta

Felipe Isaza

StudyDescription

NameOfPhysiciansReadingStudy

Consulta dermatológica

Ángela Zuluaga

StudyInstanceUID

1.3.6.1.4.1.25987.04.5404916.001.20070724.191538

StudyID 001

Patient Study Module

PatientsAge

024Y

AdditionalPatientHistory

Dolor en mano derecha

Clinical Trial Study Module

\section{Información serie}

General Series Module

SeriesDate

SeriesTime

AccessionNumber

Modality XC

InstitutionName

SeriesDescription

PerformingPhysiciansName

OperatorsName

BodyPartExamined

Protocol/Name

20070724

194001

1010

Centro Dermatológico CES Sabaneta

Mano dorsal, derecha

Pedro Díaz

Carlos Ruiz

HAND

Consulta dermatológica protocolo de fotografía médica

Figura 15. Acceso web al VL fotográfico IOD 
En la figura 15 se observa en a) la página de inicio de la aplicación web; el usuario podrá ingresar a revisar o buscar sus estudios correspondientes, gestionar sus detalles de información y salir de la aplicación. En b) se observan las series pertenecientes a un estudio dermatológico DICOM en particular, y en c) se observa un reporte general sobre una imagen dermatológica de estudio.

\section{CONCLUSIONES Y TRABAJO FUTURO}

La creación y validación de IOD DICOM en XML Schema para la representación de fotografías médicas digitales que integran información clínica y fotográfica es un claro acercamiento a la aplicación de estándares de información en especialidades médicas y, en nuestro caso, a la telemedicina. Diversos autores ${ }^{7,17}$ concluyen que la necesidad de desarrollar y aplicar estándares y guías para la implementación de sistemas en telemedicina es prioritaria. En el caso de la teledermatología, esta necesidad se aprecia en todos los aspectos de la teleconsulta dermatológica, desde la adquisición de imágenes y su resolución hasta la estructura de la información clínica enviada al teleexperto por parte del médico referente. Con el desarrollo y la publicación del VL Photographic IOD por parte de DICOM, estas necesidades se van supliendo por intermedio de un estándar de información y comunicación que nos permite buscar, visualizar y almacenar fotografías médicas digitales con su respectiva información.

Técnicamente, el desarrollo de documentos que representan objetos DICOM por medio de XML brinda un alto nivel de operabilidad, modularidad, estructuración de información y fácil transmisión por la web. Además, brinda la flexibilidad de transformar los documentos XML a otros formatos útiles, como PDF, así como "parsear" y "renderizar" documentos $\mathrm{XML}$

La relevancia de la metodología aplicada para representar objetos de información DICOM radica en su aplicabilidad para representar la totalidad del estándar DICOM, permitiendo la representación de estructuras más complejas como los reportes estructurados. Así mismo, permite interactuar con vocabularios controlados para reportar información médica reduciendo la ambigüedad de informes en formato de lenguaje natural, aumentando la precisión, claridad y el valor de los documentos clínicos ${ }^{18}$.

Dentro de los trabajos similares desarrollados a la fecha ${ }^{9,12-14}$, la creación de los XML Schemas se ha realizado con técnicas complejas patentadas que utilizan diversas herramientas de desarrollo de aplicaciones tales como Majix ${ }^{19}$, Java ${ }^{20}$ y Xerces Document Object Model Parser ${ }^{21}$, que realizan un proceso semiautomático de generación de XML Schemas a partir de conversiones especiales de la información impresa del estándar DICOM. Nuestro desarrollo fue manual, lo que permitió un claro entendimiento de las capacidades de XML Schema dentro del estándar DICOM.

Los resultados son acordes con los rasgos generales descritos por Zhao et al. ${ }^{13}$ y Lee et al. ${ }^{14}$ para la generación de documentos DICOM. Es de destacar que nuestro desarrollo modular es extensible para la creación de otros IOD y que es fácil el mantenimiento y actualización de archivos.

Para terminar, este acercamiento a la representación del estándar DICOM en XML Schema para recopilar y describir información clínica y fotográfica en telemedicina es la base para trabajos futuros en diversas áreas médicas que requieran el estudio de imágenes fotográficas. A partir de este enfoque se podrían obtener resultados en el manejo estandarizado e interoperable de imágenes provenientes de biotecnología y patología, entre las más importantes.

\section{RECONOCIMIENTOS}

El joven investigador de este proyecto fue financiado por el Instituto Colombiano para el Desarrollo de la Ciencia y la Tecnología (Colciencias), la Escuela de Ingeniería de Antioquia y la Universidad CES. Este proyecto cuenta con el apoyo del proyecto "Servicios 
basados en web para acceder a objetos DICOM", financiado por Colciencias cod. 121614-17898, la Universidad CES y la Universidad EAFIT.

Los autores desean expresar los agradecimientos al Grupo de Investigación en Bioingeniería (GIB) por su apoyo y soporte técnico y a la empresa Altova por suministrar la licencia del su producto XMLSpy.

\section{REFERENCIAS}

1. Digital imaging and communications in medicine (DICOM). Part 1: Introduction and overview [Internet]. Rosslyn: National Electrical Manufacturers Association; 2007 [acceso julio 2007]. Disponible en: http://medical.nema.org/dicom/2007/07_01pu.pdf

2. Tirado-Ramos A., Hu J. and Lee K. P. Information object definitions-based UML representation of DICOM structured reporting: a case study on transcoding DICOM to XML. J Am Med Inform Assoc. 2002; 9:6371.

3. Chartier A. DICOM (Digital imaging and communications in medicine) in dermatology. En: Wooton R. and Oakley A. (eds.). Teledermatology. London: The Royal Society of Medicine Press Limited; 2002. 205 p.

4. Digital imaging and communications in medicine (DICOM). DICOM brochure [Internet]. Rosslyn: National Electrical Manufacturers Association; 2007 [acceso julio 2007]. Disponible: http://medical.nema. org/dicom/geninfo/Brochure.pdf

5. Digital imaging and communications in medicine (DICOM). Part 3: Information Object Definitions [Internet]. Rosslyn: National Electrical Manufacturers Association; 2007 [acceso julio 2007]. Disponible en: http://medical.nema.org/dicom/2007/07_03pu.pdf

6. Digital imaging and communications in medicine (DICOM). Supplement 15: Visible light image for endoscopy, microscopy, and photography [Internet]. Rosslyn: National Electrical Manufacturers Association; 1999 [acceso julio 2007]. Disponible en: ftp:// medical.nema.org/medical/dicom/final/sup15_ft.pdf

7. Petersen R. and O'Reilly M. The development of standards in teledermatology. En: Wooton R. and Oakley A. (eds). Teledermatology. London: The Royal Society of Medicine Press Limited; 2002. p.193.

8. W3C. XML Accessibility Guidelines [Internet]. USA : W3C; 2002 [acceso julio 2007]. Disponible en http:// www.w3.org/TR/xag
9. Hu J., Lee K. P., (inventores). DICOM XML DTD/ Schema Generador. Patente Norteamericana. US 6,725,231 B2. USPTO 20-04-2004.

10. Health Level Seven HL7 [Internet]. Ann Arbor: Health Level Seven; 2007 [acceso julio 2007]. Disponible en: http://www.hl7.org.

11. Digital imaging and communications in medicine (DICOM). Strategic document [Internet]. Rosslyn: National Electrical Manufacturers Association; 2007 [acceso julio 2007]. Disponible en: http://medical. nema.org/dicom/geninfo/Strategy.pdf

12. Noumeir R. DICOM structured report document type definition. IEEE Trans Inf Technol Biomed. 2003 Dec; 7(4): 318-28

13. Zhao L., Lee K.P. and Hu J. Generating XML schemas for DICOM structured reporting templates. J Am Med Inform Assoc. 2005 Jan-Feb; 12(1): 72-83.

14. Lee K. P. and Hu J. XML schema representation of DICOM structured reporting. J Am Med Inform Assoc. 2003, 10: 213-223.

15. Falla A. M., Ortiz F. A., García A., Trefftz H. y Roldán S. Servicio basado en la web para acceder y presentar objetos persistentes DICOM. En: Memorias XVII Encuentro Nacional de Investigación Odontológica y I Reunión de la International Association for Dental Research Colombia. Bucaramanga, Colombia, Sep. 14-16 de 2006. Asociación Colombiana de Facultades de Odontología ACFO. p. 35, Sep 2006. CD-ROM ISSN 1900-3749, Sep 14-16 de 2006.

16. Digital imaging and communications in medicine (DICOM). Part 5: Data structures and encoding [Internet]. Rosslyn: National Electrical Manufacturers Association; 2007 [acceso julio 2007]. Disponible en: http://medical.nema.org/dicom/2007/07_05pu.pdf

17. Loane M. and Wootton R. A review of guidelines and standards for telemedicine. J of Telemed Telecare, 2000; 8: 63-71.

18. Hussein R., Engelmann U., Schroeter, A. and Meinzer, H.P. DICOM structured reporting: Part 1 Overview and characteristics. RadioGraphics 2004;24:891-896.

19. Tetrasix. Majix [Online]. USA, 2007. [acceso noviembre 2007]. Disponible en: www.garshol.priv.no/download/xmltools/prod/Majix.html

20. Sun Microsystems. Java. USA, 2007. [acceso noviembre 2007]. Disponible en: www.java.com/en/

21. The Apache Software Foundation. USA, 2007. [acceso noviembre 2007]. Disponible en: http://xerces. apache.org/ 